\title{
Clase, género y movilidad social: articulaciones conceptuales para el estudio de la reproducción social
}

\section{Class, gender and social mobility: conceptual articulations for the study of social reproduction}

Sofía Vanoli Imperiale*

Resumen: Este artículo dispone una discusión teórica sobre las posibilidades de conjunción del análisis de género y el análisis de clase para el estudio sociológico de la movilidad y reproducción social. Repasando una tradición que ha tenido como resultado la separación de los campos de estudio y la ausencia del género como factor relevante en los análisis clásicos de movilidad social, se presentan las posibilidades para un andamiaje conceptual que pueda sostener estudios empíricos capaces de articular el género y el origen social como fuentes explicativas de la desigualdad social; esto alimentado, a su vez, por la proliferación en las últimas décadas en Europa y en América Latina de investigaciones sobre movilidad que, al incluir como unidad de análisis tanto a varones como a mujeres, advierten que las conclusiones sobre los volúmenes y las características de la movilidad de clase se complejizan.

Palabras clave: Desigualdad social. Género. Análisis de clase.

\begin{abstract}
This article provides a theoretical discussion on the possibilities of conjunction of gender analysis and class analysis for the sociological study of mobility and social reproduction. Reviewing a tradition that has resulted in the separation of those fields and the lack of consideration of gender as a relevant factor in classical studies of social mobility, it presents the possibilities for a conceptual net capable of supporting empirical studies that articulate gender and social origin as explanatory sources of social inequality. That, moreover, has been fuelled by the proliferation in recent decades in Europe and Latin America of researches on mobility that, when including both men and women, convey that conclusions about social reproduction become more complex.
\end{abstract}

Keywords: Social inequality. Gender. Class analysis.

Recebido em: 26/07/2020. Aceito em: 24/06/2021.

*Estudiante de maestría en Sociología y licenciada en Sociología por la Facultad de Ciencias Sociales de la Universidad de la República (Uruguay). E-mail: sofia.vanoli@cienciassociales.edu.uy.

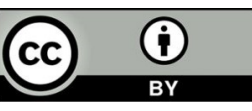




\section{Introducción}

Este trabajo pretende repasar y contribuir al intercambio conceptual entre el análisis de género y el análisis de clase que se ha derivado de las críticas que a finales del siglo XX, principalmente desde los feminismos, se le han realizado a los estudios clásicos de movilidad social, que sistemáticamente han dejado por fuera de sus diseños de investigación a las mujeres.

Se busca problematizar con este esfuerzo de revisión teórica y articulación conceptual la distancia con la que se han desarrollado en el campo de la sociología los estudios de género y los estudios de clase. Se argumenta así que no son fuentes de desigualdad que actúen de forma autónoma sino que se encuentran histórica y sistemáticamente imbricadas, y la conceptualización que se realice de ese modo de imbricación propone diferentes desafíos teóricos y metodológicos para el estudio de la desigualdad social.

Para presentar esa problematización, se trazará el siguiente recorrido: en primer lugar se definirá la movilidad social y su vinculación con otros conceptos como la meritocracia y la igualdad de oportunidades, a la vez que se repasarán sus principales hitos como campo de investigación sociológica en Europa, Estados Unidos y América Latina; de forma similar, en segundo lugar se introducirán los conceptos articuladores del análisis de género, las interconexiones que desde ese campo se fueron esbozando entre género y clase social, así como las críticas que desde esa perspectiva le fueron realizadas a los estudios tradicionales de movilidad social; para finalmente recorrer las discusiones sobre la ausencia de las mujeres en los estudios de movilidad social y los desafíos teóricos y metodológicos que emergen con su inclusión en las investigaciones contemporáneas.

Este es, por lo tanto, un artículo descriptivo de discusión conceptual, generado a partir de una metodología basada en la revisión teórica y bibliográfica de los conceptos nucleares en los campos del análisis de género y el análisis de movilidad social de clase; así como en la observación de los marcos teóricos que sustentan algunas de las investigaciones clásicas sobre movilidad social (aquellas realizadas principalmente en Europa durante la segunda mitad del siglo XX), así como otras más recientes (principalmente investigaciones latinoamericanas de estas primeras décadas del siglo XXI), prestando atención especial a la inclusión u omisión del concepto de género en sus postulados.

Este esfuerzo de articulación conceptual forma parte del proceso de realización de una tesis por parte de la autora para obtener el grado de magíster en sociología.

\section{Los estudios de movilidad social y sus principales énfasis}

La movilidad social implica el cambio en la posición en que se encuentra un individuo en la estructura social (ya sea respecto a sus antecesores o respecto a sí mismo a lo largo de su vida), y constituye un fenómeno fundamentalmente moderno. En sociedades premodernas ese movimiento era casi imposible en función de la estricta herencia del origen a partir de la cual se reproducía la sociedad. Difícilmente, por ejemplo, un hijo o hija de campesinos de la época feudal imaginara, intentara o lograra otro destino que no fuera el que le dio origen. Las revoluciones modernas desde la postulación de una libertad e igualdad formal y la consolidación de un modo de producción que puso en relación a individuos libres de vender su fuerza de trabajo no sólo hicieron posible la movilidad entre posiciones sociales sino que la legitimaron como una aspiración vital. El sujeto moderno está conminado a moverse para lograr bienestar y ascenso social. 
Esa forma de entender la movilidad social se relaciona íntimamente con un relato que la asocia a la modernización y al desarrollo, y cobra sentido al considerar la puja ideológica que atraviesa y define el siglo XX entre el capitalismo en un estado avanzado de producción y consumo y la propuesta política de un marxismo que vivió su ensayo real con la creación de la Unión Soviética. Mientras la crítica radical del socialismo se funda en la desigualdad de clases y en el carácter intrínseco de esa desigualdad para el sistema capitalista, la respuesta liberal de esa crítica encuentra en una movilidad social fundada en la meritocracia la herramienta conceptual para correr la discusión desde la igualdad de condiciones a la igualdad de oportunidades.

El concepto de meritocracia, por lo tanto, desplaza el problema de la desigualdad desde el reparto desigual de bienestar al modo a través del cual se realiza ese reparto, enfatizando una asignación de recompensas basada en el mérito y no la herencia. Gayo y Méndez (2007, p. 123) indican respecto a eso que "bajo el supuesto de que las sociedades son inequitativas, se estudia la forma de asignación de posiciones o, si se quiere, de recursos, dejando atrás los debates que contraponían las sociedades igualitarias a las que no lo eran, y sustituyéndolos por la meritocracia y la carencia de ella". Esto implica que una sociedad será justa si se funda en el mérito, con independencia de si es o no igualitaria. La meta ya no es la igualdad de condiciones, es decir, la asignación equitativa de recursos, sino la igualdad de oportunidades, esto es, que todos los individuos tengan las mismas chances de obtener mérito para lograr determinada posición social. Que las posiciones sociales se asignen en función del mérito y no la herencia supone empíricamente que origen y posición social no se encuentren asociados (CARABAÑA, 1980). Es decir, que todos los individuos tengan las mismas oportunidades de llegar a cada posición social, sin importar el origen del que partan. La movilidad social se convierte así en el referente empírico para la contrastación de la meritocracia.

Como programa de investigación sociológica los estudios de movilidad social comenzaron a desarrollarse con mayor sistematicidad y precisión técnica terminada la Segunda Guerra Mundial. Las razones de ese auge se encuentran fundadas en las condiciones geopolíticas que trazaban un mundo dividido por dos grandes formas de organización socioeconómica. Desde el sustrato teórico de cada uno de esos mundos podía ser formulada, a su vez, una hipótesis vinculada a la estructura de clases y la movilidad social. Mientras el marxismo auguraba para las sociedades capitalistas una progresiva proletarización de su estructura de clases, enraizada en el mecanismo de explotación; las teorías liberales apoyadas en el funcionalismo mantenían su propio set de predicciones para las sociedades industrializadas: el incremento progresivo de las tasas de movilidad ascendente y la igualdad de oportunidades. ${ }^{1}$ En ese marco de disputa teórica y política los análisis de movilidad resultaban una fuente de evidencia para apoyar una u otra predicción.

La sucesión de investigaciones sobre estratificación y movilidad social que se desarrollaron desde mediados del siglo XX puede ser dividida en tres grandes generaciones que se distinguen tanto respecto a los intereses teóricos que persiguieron, y consecuentemente respecto a sus principales hipótesis, como a los métodos de análisis que utilizaron. El mayor punto de contacto, no obstante, fue el énfasis en los diseños comparativos entre países con diferentes formas de organización política y en distintos niveles de industrialización, de modo de poder capturar la relación entre el avance industrial moderno y el grado de apertura de una sociedad, que suponía el interés primigenio de los estudios de movilidad.

\footnotetext{
${ }^{1}$ Un desarrollo detallado de autores e implicaciones teóricas de ambas hipótesis puede ser encontrado en el capítulo inicial de The constant flux (ERIKSON; GOLDTHORPE, 1993).
} 
Ganzeboom, Treiman y Ultee (1991) identifican una primera generación de estudios de movilidad protagonizada, entre otros, por Glass, Lipset, Bendix y Miller, que ubicados fundamentalmente en Europa desde 1950 y guiados en su mayoría por la hipótesis liberal de que el avance tecnológico implicaría un reparto más óptimo de las recompensas en función del mérito propusieron investigaciones comparativas a partir de grandes encuestas nacionales y una forma de observar el fenómeno desde el análisis de tablas de movilidad intergeneracional, esto es, tablas de contingencia entre el origen social de los encuestados y su posición social al momento de ser consultados.

La pregunta comparativa que dio unidad a esta primera generación fue, inicialmente, si los patrones de movilidad se diferenciaban entre los países, con la asunción frecuente de que Estados Unidos, como nueva nación, ofrecería una apertura mayor (GANZEBOOM; TREIMAN; ULTEE, 1991). Una segunda hipótesis frecuente asociaba mayores niveles de movilidad en sociedades industrializadas y patrones más rígidos en aquellas no industrializadas, mientras una tercera cuestión relevante fue la conexión entre la estructura política de una nación y sus niveles de movilidad intergeneracional.

La potencia de las investigaciones que componen esta primera generación de estudios para responder sus preguntas centrales fue limitada por el poco refinamiento estadístico de sus formas de análisis. Así, el procesamiento de las tablas de movilidad frecuentemente se limitó a observar sus diferentes porcentualizaciones, obteniendo así tasas absolutas de movilidad, porcentajes de entrada y porcentajes de salida de las posiciones sociales. A nivel inferencial la hipótesis de contraste principal fue la de movilidad perfecta (operacionalizada como la independencia estadística entre origen y destino), cuya constante refutación poco podía decir sobre la especificidad de los regímenes de movilidad. Pese a estas limitaciones metodológicas, varios investigadores reconocieron que las tasas de movilidad estaban influenciadas por los cambios estructurales que se podían observar en los marginales de las tablas origen destino, y propusieron formas de aislar ese efecto para observar más claramente los patrones de movilidad.

La segunda generación de estudios sobre movilidad social implicó un corrimiento tanto de las hipótesis de interés como de las formas de abordaje metodológico. Cronológicamente se ubica a fines de la década de los sesenta y puede citarse como estudio protagónico el publicado por Blau y Duncan (1978) en 1967 sobre Estados Unidos. Las dos grandes innovaciones de ese estudio fueron la utilización de una escala de estatus ocupacional (ISEI) que al ser una variable continua permitió, a su vez, el uso de técnicas de análisis de regresión como el path analysis. Esto supuso no sólo el abandono de las tablas de movilidad sino la emergencia de nuevas hipótesis vinculadas fundamentalmente a estudiar el grado de correlación entre el origen y el destino social y, a su vez, en qué medida ese efecto era directo y en qué medida estaba mediado por el logro educativo y la ocupación inicial.

La tercera generación ${ }^{2}$, identificable desde mediados de los setenta, se caracteriza por la vuelta al análisis de las tablas de movilidad mediante un refinamiento muy claro de los procedimientos estadísticos para hacerlo y una mejora en el relevamiento de datos a escala internacional. Esto permitió la complejización de las hipótesis originarias de Glass a través de los desarrollos iniciales de Goodman en 1965, que estimuló el surgimiento de una nueva hipótesis:

\footnotetext{
${ }^{2}$ Pese a que en el artículo referido Ganzeboom, Treiman y Ultee (1991) identifican esta tercera generación como la última, en un artículo más reciente (2000) reconocen una cuarta generación de estudios de movilidad social, aún incipiente, marcada por la realización de nuevas investigaciones internacionales comparativas, un mayor énfasis en los arreglos institucionales de las naciones y la inclusión de otras técnicas de análisis como la historia de eventos.
} 
la de cuasi-independencia, y la creación de los modelos loglineales; lo que motivó a su vez la construcción de modelos posteriores más complejos, como el modelo topológico de Hauser en 1978 (basado en la consideración de diferentes zonas de asociación en la tabla de contingencia, de forma de mapear los efectos de las variables sobre la distribución obtenida) o los modelos de fronteras y distancias de Goodman.

En términos de análisis, el uso de los modelos loglineales en lugar de la utilización de técnicas de regresión permitió separar más claramente la movilidad neta de los cambios en los marginales, que había sido una ambición no resuelta de la primera generación. A su vez, ese tipo de modelos hace posible tratar una relación bivariada como un patrón multidimensional (HOUT, 1983), por lo que permite el contraste de hipótesis que den más información sobre el vínculo entre origen y destino que la independencia estadística. En términos teóricos, la revitalización del análisis a partir de tablas de movilidad implicó una defensa de la importancia de la clase social para entender la reproducción de la desigualdad.

No hay duda en identificar a Goldthorpe $(1987,2010)$ como el autor protagónico de esta tercera generación, tanto por haber sido director del mayor proyecto comparativo internacional de esa época (el proyecto CASMIN, que comparó los patrones de movilidad en 13 naciones industrializadas), como por haber formalizado la potencia de los modelos loglineales para los estudios de movilidad social en la distinción conceptual entre movilidad absoluta y movilidad relativa.

Para el autor la movilidad absoluta supone el estudio de las tasas de movilidad incluyendo la movilidad estructural, esto es, el cambio en el tamaño de las clases entre las distribuciones de origen y las distribuciones de destino, mientras la movilidad relativa neutraliza ese efecto mediante el cálculo y comparación de las probabilidades condicionales de encontrarse en determinada clase habiendo tenido origen en otra. Este último tipo de movilidad, por lo tanto, permite una observación más precisa sobre los vínculos intrínsecos entre las posiciones sociales.

Aislar el efecto de los marginales, además, implica una segunda ventaja: hacer posible la comparación de los patrones de movilidad entre naciones con diferencias en su estructura social. Fue justamente ese punto el que supuso la elaboración y contrastación de una de las hipótesis más conocidas de Goldthorpe, desarrollada en su libro de 1993 junto a Erikson: The constant flux, donde frente a la teoría liberal del industrialismo, que hipotetizaba altas tasas de movilidad con predominio de la movilidad ascendente, mayor equidad en las chances de movilidad y el incremento a través del tiempo de la igualdad de oportunidades, concluyen contrariamente que la movilidad relativa -o fluidez social- es similar entre periodos y naciones industrializadas, pese a las variaciones que pueden observarse en las tasas absolutas de movilidad. En términos generales, la pauta de movilidad y herencia en las sociedades occidentales se mantiene a través del tiempo y puede especificarse a partir de las siguientes regularidades:

La inmovilidad se da sobre todo entre los campesinos, seguidos de la pequeña burguesía y la clase de servicio. La inmovilidad en la cima de la jerarquía socioeconómica...es mayor que en su punto más bajo, en la clase obrera. La movilidad es más probable en el punto "medio" de la jerarquía socioeconómica (o estructura de clases) que en su punto más alto. La movilidad de corto alcance es más frecuente que la movilidad de largo alcance. (KURZ; MÜELLER, citado en CROMPTON, 1994, p. 95).

Ese énfasis en la comparación internacional de los patrones de movilidad, a su vez, supuso la consolidación del esquema de clases EGP (ERIKSON; GOLDTHORPE; PORTOCARERO, 1979) para la medición de la estructura social y la estandarización de un conjunto de tesis, además de la 
fluidez social, para contrastar en los patrones de movilidad social, como el cierre de la cumbre y la movilidad de corta distancia.

¿Qué sucedía mientras tanto en Latinoamérica respecto a ese tipo de estudios? Los análisis de movilidad social en los países latinoamericanos surgen vinculados al concepto de desarrollo y, consecuentemente, a la teoría de la modernización (GERMANI, 1969). Esta perspectiva identificó dentro del componente social del proceso de modernización un aumento de la movilidad social basada en la meritocracia. En ese marco puede comprenderse que acompañado a sus planteos se hayan realizado estudios específicos sobre movilidad social en Latinoamérica, como el estudio propio de Germani sobre "La movilidad social en Argentina", publicado en 1963 en el libro de Lipset y Bendix -lo que redunda en su afiliación con las hipótesis conceptuales de esa primera generación de estudios sobre movilidad social- y el análisis de Iutaka (1962) sobre "Estratificación social y oportunidades educacionales en tres metrópoli latinoamericanas", analizando los volúmenes de movilidad social en Buenos Aires, Montevideo y San Pablo.

Luego de un freno identificado entre la década de los ochenta y finales de siglo, donde en el marco de la crisis de la deuda y el decrecimiento económico (FRANCO; LEÓN; ATRIA, 2007) las ciencias sociales abandonaron los estudios de estratificación y movilidad social para enfocarse en el concepto de pobreza, desde los 2000 se ha observado un resurgimiento de los estudios de movilidad social en los países de América Latina. ${ }^{3}$

\section{Sobre el concepto de género como categoría analítica}

El concepto de género, que demostró canalizar un gran potencial político hacia finales del siglo XX de la mano principalmente de los feminismos, fue concebido inicialmente por el sexólogo neozelandés John Money en la década de los 70. Especializado en el estudio de niños intersexuales, para los que promovía una cirugía de asignación de sexo temprana, Money desplazó de la lingüística el término género para referirse a la masculinización o feminización de esos niños, luego de operados, mediante un proceso de aprendizaje sociocultural (DI SEGNI, 2013).

Rápidamente trasladado a la esfera política, el término le permitió a los feminismos de segunda ola ${ }^{4}$ desarraigar las diferencias entre mujeres y varones de la biología, dándole a ambas categorías la materialidad social que Simone De Beauvoir (1949) ya había argumentado en El segundo sexo. El término permitió a su vez otros derivados conceptuales fundamentales como el de sistema sexo/género que, expuesto por primera vez por la antropóloga estadounidense Gayle Rubin (1986), refiere al "conjunto de disposiciones por el que una sociedad transforma la sexualidad biológica en productos de la actividad humana" (RUBIN, 1986, p.3). Esa forma de entender las diferencias entre mujeres y varones hizo posible la legitimación conceptual de varios puntos que formaban parte del programa político de los feminismos: (a) considerar las diferencias entre lo

\footnotetext{
${ }^{3}$ De esa etapa, que aún se encuentra en proceso, pueden destacarse como ejemplo las siguientes investigaciones: para Argentina Jorrat (2000, 2008, 2014 y otros), Kessler y Espinoza (2007), Pla (2012), Pla y Rodríguez de la Fuente (2016) y Dalle (2015); para Chile Torche y Wormald (2004) y Espinoza, Barozet y Méndez (2013); para Brasil Scalon (1997) y Do Valle Silva (2007); para México Cortés y Escobar (2007) y Solís y Cortés (2009); para Uruguay Boado (2008, 2010, 2013); así como estudios comparados como el coordinado por Boado y Solís (2016) sobre Argentina, Brasil, Chile, Perú, México y Uruguay.

${ }^{4}$ La segunda ola del feminismo refiere al conjunto de reflexiones teórico-políticas que desde los años 60 profundizaron las demandas del movimiento feminista (que en su primera ola de principios de siglo XX se había centrado en la conquista de derechos ciudadanos como el sufragio), contemplando no sólo las desigualdades referidas a la esfera pública sino las raíces de la dominación patriarcal en la división sexual del trabajo y en la construcción socio-cultural de las diferencias entre varones y mujeres.
} 
masculino y lo femenino desde un proceso de socialización que dispone para varones y mujeres un aprendizaje especial de características, símbolos y roles a partir de cierta composición biológica diferencial de sus cuerpos (b) postular que en ese sentido las diferencias no son biológicas sino biologizadas mediante la vinculación entre el sexo y el género ${ }^{5}$ (c) entender consecuentemente las relaciones sociales entre varones y mujeres como parte de un mismo sistema jerarquizado de poder construido y reproducido intergeneracionalmente a través de la socialización de género.

Además de ser utilizado en el terreno político, el concepto de género ha ingresado en el ámbito académico de las ciencias sociales edificando campos específicos en el seno de las diferentes disciplinas así como argumentando la necesidad de su incursión transversal en otros campos. Probablemente uno de los esfuerzos más conocidos de ese tipo de argumentación sea el artículo "El género: una categoría útil para el análisis histórico" de Joan Scott (1996), publicado por primera vez en 1986. En él la historiadora estadounidense justifica la necesidad de utilizar el género como categoría analítica a la vez que debate sobre el potencial explicativo de las diferentes perspectivas en torno al concepto: la visión feminista abocada a entender los orígenes del patriarcado, la tradición marxista que funda la opresión de género a partir de su peso en el sostenimiento de las relaciones de producción, y las escuelas con base en el psicoanálisis dispuestas a estudiar la conformación de las identidades de género.

Pese a las diferencias entre esos enfoques, que analiza críticamente, Scott sostiene que la consideración del género como categoría analítica es ineludible para el análisis social, y esto porque no sólo supone la integración de las mujeres como unidad de análisis tradicionalmente invisibilizada en el estudio de los procesos históricos y de los fenómenos sociales, sino porque desde una perspectiva relacional entiende que si se omiten las consideraciones de género ni siquiera es posible un análisis confiable de los procesos para los varones. "Las estudiosas feministas pronto indicaron que el estudio de las mujeres no sólo alumbraría temas nuevos, sino que forzaría también una reconsideración crítica de las premisas y normas de la obra académica existente" (SCOTT, 1996, p. 3). En ese sentido los estudios de género a diferencia de los estudios sobre mujeres, enfatizan primordialmente "que la información sobre las mujeres es necesariamente información sobre los hombres, que un estudio implica a otro" (SCOTT, 1996, p. 6). Esto es el carácter relacional del género, como un sistema de relaciones de interdependencia.

\section{Articulaciones teóricas entre género y clase}

El énfasis visto anteriormente en el uso analítico del concepto de género hacia finales del siglo XX para explicar los procesos sociales pronto motivó el debate sobre su vínculo con otros conceptos que cargaban una historia más larga de protagonismo en ese tipo de investigaciones. Principalmente con el concepto de clase. En la mayoría de los casos esos debates fueron directamente con el marxismo, erguido hasta el momento como la crítica más radical al estado de las cosas, y cuna conceptual de muchas de las autoras feministas que comenzaban a trabajar con mayor sistematicidad el concepto de género. Si la clase constituía un eje fundante de la desigualdad social y su reproducción, pero también lo era el género, ¿cómo se podían articular los dos conceptos?

\footnotetext{
${ }^{5}$ Cabe destacar que un posterior desarrollo crítico al respecto en el seno del feminismo condujo a Judith Butler (2007, 2010) a desmantelar ese mismo sistema sexo/género acusándolo de reafirmar la biologización que pretendía denunciar. En sus textos, Butler se esfuerza por argumentar que no sólo el género tiene una materialidad socio-cultural sino también el sexo que, propuesto como biológico y prediscursivo, se inscribe en los cuerpos por la acción performativa del género. Al respecto puede verse El género en disputa. El feminismo y la subversión de la identidad (2007) o Cuerpos que importan. Sobre los límites materiales y discursivos del sexo (2010).
} 
¿Se fundan en el mismo criterio de constitución de la estructura social o responden a raíces ontológicamente diferenciadas?

Desde los desarrollos feministas aún identificados con el marxismo más canónico la respuesta a esas preguntas no podía estar de otra manera que no fuera atada a alguna explicación material de la constitución social. En el andamiaje teórico de esta perspectiva es recurrente encontrar referencias a Engels en El origen de la familia, la propiedad privada y el Estado (publicado por primera vez en 1884), donde construye el vínculo entre la privatización de la propiedad y el quiebre del derecho materno ${ }^{6}$. Allí se identifica al género y a la clase como fuentes de desigualdad que surgen de la misma raíz: la propiedad privada. Son dos formas de considerar un único mecanismo de distinción, un antagonismo fundamental. Engels cita a Marx (ENGELS, 2006, p.72) al decir que la primera división de trabajo fue entre hombres y mujeres, y le agrega que "el primer antagonismo de clases que apareció en la historia coincide con el desarrollo del antagonismo entre el hombre y la mujer en la monogamia; y la primera opresión de clases, con la del sexo femenino por el masculino". Si tanto el género como la clase son fuentes de desigualdad -esto es, clasifican a los individuos en grupos en los que el bienestar no se distribuye de igual manera-, para el marxismo son expresiones fundadas por la misma escisión fundamental, la posesión de la propiedad.

Pese a ese reconocimiento central de la imbricación entre género y clase como expresiones del mismo proceso, el análisis feminista ha sido muy crítico respecto a la ausencia del trabajo no remunerado que principalmente las mujeres realizan en la esfera doméstica en la sofisticada articulación que Marx realiza sobre la creación de valor y sobre los elementos necesarios para la reproducción de la fuerza de trabajo7.

Además, el enfoque marxista respecto al género también ha sido discutido desde una perspectiva más general, de carácter ontológico pero con claras consecuencias para el planteo teórico y político. A esto se refiere Scott al identificar en el feminismo marxista el problema de tener que fundar siempre la estructura social en las relaciones de producción, lo que de alguna manera hace difusa la especificidad del género en la reproducción de la desigualdad.

Las feministas marxistas tienen una perspectiva más histórica, guiadas como están por una teoría de la historia. Pero cualesquiera que hayan sido las

\footnotetext{
${ }^{6}$ Explica Engels que mientras no existió la propiedad privada como tal y el modo de producción preponderante fue el que junto a Marx denominaron comunismo primitivo, la reproducción era guiada por lo que llama el derecho materno. Esto implica que, dado que las trabas sexuales eran mínimas y el matrimonio podía ser grupal, sólo era posible asegurarse la consanguineidad de los hijos con sus madres, lo que convertía ese tipo filiatorio en la estructura de parentesco principal. Esa situación no se transformó hasta que el surgimiento de métodos de producción como la cría de ganado hizo posible la generación de riqueza y la acumulación, que a su vez se fue convirtiendo en propiedad particular, haciendo emerger una nueva forma de orden social protagonizado por la familia monogámica. "A medida que iban en aumento, las riquezas daban al hombre una posición en la familia más importante que a la mujer y hacían que naciera en él la idea de valerse de esta ventaja para modificar en provecho de sus hijos el orden de herencia establecido. Pero esto no podía hacerse mientras permaneciese vigente la filiación según el derecho materno. Éste tenía que ser abolido, y lo fue” (ENGELS, 2006, p. 63). Engels reconoce así en la abolición del derecho materno la gran derrota de las mujeres: "de ninguna manera la monogamia aparece en la historia como una reconciliación entre el hombre y la mujer, y menos aún como la forma más elevada de matrimonio. Al contrario, entra en escena bajo la forma de la esclavización de un sexo por el otro, como la proclamación de un conflicto entre los sexos, desconocido hasta entonces en la prehistoria" (ENGELS, 2006, p. 72).

${ }^{7}$ Pueden consultarse al respecto los desarrollos de Silvia Federici en Calibán y la bruja: mujeres, cuerpo y acumulación originaria (2015) o en artículos como "La reproducción de la fuerza de trabajo en la economía global y la inacabada revolución feminista" (2008), donde expresa que "el eje central de esta crítica lo articula la afirmación de que el análisis que Marx hizo del capitalismo se ha visto lastrado por su incapacidad de concebir el trabajo productor de valor de ningún otro modo que no sea la producción de mercancías y su consecuente ceguera sobre la importancia del trabajo no asalariado de las mujeres en el proceso de acumulación capitalista" (p. 32).
} 
variaciones y adaptaciones, la exigencia auto impuesta de que debería haber una explicación "material” para el género, ha limitado, o al menos retardado, el desarrollo de nuevas líneas de análisis. (...) En la discusión marxista ortodoxa de los modos de producción, la explicación de los orígenes y cambios en los sistemas de género se plantea al margen de la división sexual del trabajo. Al final, familias, hogares y sexualidad son todos productos de modos de producción cambiantes. (SCOTT, 1996, p. 11).

De alguna manera la cuestión de fondo es rastreable en otros debates como el contrapunto entre Nancy Fraser y Judith Butler (2000) y refiere a si las desigualdades de género y de clase tienen sus raíces en esferas diferentes de la constitución social o son dimensiones de un mismo proceso de estructuración de lo social. En sus consecuencias políticas y en palabras de Fraser y Butler, la respuesta a esa cuestión implica diferentes ponderaciones y diseños de políticas de reconocimiento y políticas de redistribución.

Rosemary Crompton (2003) revisa ese debate en una discusión específica sobre articulaciones entre género y clase que a le vez critica lo que llama el giro cultural posmoderno. La autora distingue en su rastreo teórico sobre la temática un abordaje dual que enfatiza la separación de la esfera económica y cultural y un abordaje unitario que se niega a identificar una separación entre esos ámbitos, y diagnostica como resultado de este último enfoque una culturización de los problemas sociales que eclipsa la importancia del análisis de clase. Más allá de las derivas de esa discusión que está vinculada además con el desarrollo del posestructuralismo, lo que es de interés principalmente para este estudio es la reflexión de Crompton respecto a las consecuencias diferenciales de esas perspectivas sobre la posibilidad de articular clase y género en investigaciones empíricas:

Si la cultura y la economía se ven como sistemas duales (aunque estrechamente relacionados) o como formando una totalidad, tiene implicaciones fundamentales para la investigación empírica. Un enfoque de sistema dual para la cultura/economía es perfectamente compatible con los enfoques cuantitativos para la estratificación, orientados a variables, que pueden incorporar tanto variables culturales como económicas. También es compatible con enfoques sociológicos y antropológicos relativamente ortodoxos.

Un enfoque unitario de la cultura y la economía está más restringido en sus métodos. Está claro que los métodos “orientados a variables" no serían apropiados. Es probable que los métodos empleados deban ser de grano fino, etnográficos e históricos. (CROMPTON, 2003, p. 18; traducción propia)

Su opción, por lo tanto, apunta a un "dualismo de perspectiva" que permita las explicaciones causales referidas a la clase y al género y evite la colonización conceptual de uno por el otro, en tanto "se puede argumentar que un enfoque de 'economía cultural' con respecto a la investigación sobre género y empleo ha servido para oscurecer las continuas desigualdades de género en el empleo que deberían ser centrales, más que periféricas, para los debates entre género y clase" (CROMPTON, 2003, p. 18; traducción propia).

La propuesta entonces, en la que se apoya este trabajo, no supone necesariamente la defensa de una separación ontológica y ahistórica entre género y clase, sino el abordaje de la desigualdad social desde un dualismo que es de carácter principalmente metodológico y que se apoya en la diferenciación de las dimensiones justamente para la exploración y explicación de las interconexiones que se manifiestan en su forma actual, con la asunción como telón de fondo de un proceso de desarrollo originario esencialmente indistinguible. 
Esa perspectiva construye un espacio de acción para el andar separado de la teoría de género y del análisis de clase pero también para la vinculación entre ambos y su consideración conjunta. En tal sentido, se puede encontrar en Erik Olin Wright (2000) una enumeración de algunas formas que puede tomar la articulación entre género y clase en el estudio de la desigualdad social: desde su afectación recíproca, en la consideración del género como un mecanismo que reparte a los individuos en posiciones sociales diferentes, como un vínculo que media la posición de clase, así como la interacción entre género y clase en la determinación de resultados.

El autor explica para ejemplificar el primer caso, en el que se considera que el género y la clase se afectan recíprocamente, que es probable que ciertos tipos de posición social sólo existan en virtud de las relaciones de género, como es el caso de los servicios domésticos. "Si las relaciones de género fueran dramáticamente más igualitarias, esos mismos trabajos podrían no existir" (WRIGHT, 2000, p. 119; traducción propia). A la inversa, el autor advierte que las relaciones de clase tienen un impacto sobre el género: "las demandas competitivas y de alta presión de muchas ocupaciones gerenciales y profesionales ayudan a reforzar un tipo específico de relaciones domésticas de género en el que las amas de casa están disponibles para manejar los asuntos personales de sus esposos" (WRIGHT, 2000, p. 120; traducción propia).

El siguiente caso de interconexión entre ambos conceptos, el género como un mecanismo de distribución de los individuos en posiciones de clase, es incluso más sencillo de reconocer. Wright (2000) identifica dos factores de reflexión teórica en las ciencias sociales que explican los procesos a través de los que el género se relaciona con el logro de destinos sociales desiguales: por un lado la socialización generizada, que modela las aspiraciones y las habilidades de varones y mujeres, y por otro lado las formas de discriminación y la inequidad que afecta directa o indirectamente las oportunidades de varones y mujeres de acceder a determinadas ocupaciones. Ambos sostienen el proceso frecuentemente denominado como segregación ocupacional de género.

El género como mediador de la ubicación de clase tiene que ver con la forma que Wright (2000) propondrá para clasificar a los individuos en posiciones sociales, vinculada no sólo con la asignación directa a través de las ocupaciones de cada sujeto sino también con la asignación indirecta mediante la consideración de mecanismos de mediación relacionados con las redes de parentesco, donde adquiere protagonismo el género.

Finalmente, Wright (2000) reconoce que el género y la clase se vinculan como factores que entran en interacción en la determinación de otros resultados. Además de actuar como mecanismos independientes, la interacción entre la clase y el género produce especificaciones en el logro de ciertos resultados como el nivel educativo, el ingreso u otras variables dependientes de interés. La tarea del análisis de clase en este punto, destaca el autor, es lograr identificar los efectos que se pueden atribuir sólo al género, sólo a la clase y a la interacción entre ambos.

Ese tipo de reflexiones aparecen hacia finales de un siglo que en el campo de los estudios de movilidad social fue testigo de una fructífera disputa conceptual sobre la forma y las implicancias de integrar a las mujeres (o en su versión relacional considerar el género) en programas de investigación que sistemáticamente las había dejado fuera. En el siguiente apartado se presenta un sucinto recorrido al respecto.

\section{La ausencia de las mujeres en los estudios de movilidad social y sus debates}

Gran parte de la acumulación teórica sobre la articulación entre la clase y el género presentada antes fue alimentada desde intercambios en torno a la movilidad social. Es que 
durante las últimas décadas del siglo XX se mantuvo una discusión entre investigadores del campo y académicas que desde el feminismo denunciaron la ausencia de análisis sobre las mujeres en los diseños de los estudios de movilidad social tradicionales (ACKER, 1973; DELPHY, 1981; STANWORTH, 1984).

La crítica fue principalmente dirigida a Goldthorpe y fundamentalmente a su forma de construcción de las clases sociales, que sólo utilizaba la ocupación de los varones jefes de hogar y de sus padres. Ese tipo de diseño se denominó el enfoque convencional y, de acuerdo a los debates que suscitó, se sostiene sobre tres presupuestos: a) que la unidad de análisis adecuada para los estudios de clase son los hogares, que a la vez se suponen nucleares y heterosexuales b) que dentro del hogar los intereses y las oportunidades de vida son homogéneas y c) que la posición social de los varones es preeminente y marca la situación de clase del hogar, en la que no se reconoce influencia posible de la posición ocupacional de la mujer.

Ante la crítica a su enfoque, Goldthorpe se defendió inicialmente mediante tres argumentos que sostenían los presupuestos detallados: que las mujeres tienen una baja tasa de participación económica, lo que dificulta su ubicación en una posición de clase, que el aumento de la tasa de participación se debe fundamentalmente al empleo a tiempo parcial, por lo que su ocupación suele ser menos destacada que el trabajo a tiempo completo de los varones y más intermitente, la baja frecuencia de los matrimonios entre clases y la asociación entre la condición de actividad de las mujeres y las posiciones de sus maridos (GOLDTHORPE, 1983; ABBOTT; PAYNE, 2005). De alguna manera se generaba una paradoja en la que el propio reconocimiento de la desigualdad entre varones y mujeres al interior de los hogares fundaba la exclusión de las mujeres de sus análisis de clase (SALIDO CORTÉS, 1996), por lo que de acuerdo a Goldthorpe toda consideración de género como fuente de desigualdad social quedaba por fuera de los objetivos del análisis de clase.

Esa respuesta, sin embargo, no llevó a que las críticas desde el feminismo cesaran. Si se observan los dos frentes esgrimidos por el autor para defender su enfoque puede reconocerse una proposición de carácter más empírico: la tasa de participación laboral femenina es baja, parcial e intermitente; y una proposición de carácter más teórico: las desigualdades ente varones y mujeres no tienen que ver con la estratificación social y deben por lo tanto estudiarse desde otro campo. Por un lado el aumento sostenido de la participación de las mujeres en el mercado laboral y la emergencia de nuevas configuraciones familiares ha desestimado la importancia del argumento más empírico, y ha implicado nuevos desafíos que también soportan la refutación del argumento más teórico, ya duramente criticado por los debates feministas sobre la articulación indisoluble entre clase y género. Apuntando a eso, Michelle Stanworth (1984, p. 159; traducción propia), en un artículo que denominó "Women and class analysis: a reply to John Goldthorpe", mantuvo que "el enfoque convencional defendido por Goldthorpe oscurece el grado en que la experiencia de clase de las esposas difiere de la de los esposos, e ignora hasta qué punto las desigualdades que dividen a las mujeres y los hombres son en sí mismas el resultado del funcionamiento del sistema de clases".

La crítica por lo tanto sostiene que lejos de quedar por fuera del análisis de clase, las desigualdades entre varones y mujeres tienen que ver con la forma en que se reproduce la estructura social, tanto desde su efecto diferencial sobre la salida al mercado laboral y sus condiciones, influidas por la carga desigual que supone la división sexual del trabajo ${ }^{8}$ al interior

${ }^{8}$ Con división sexual del trabajo se hace referencia a uno de los conceptos que edifican la reflexión en el seno de la segunda ola del feminismo y apunta a teorizar e historizar la diferenciación en la carga de trabajo, los roles y las tareas asignadas a mujeres y varones en base a su género, que construye una división jerarquizada entre esferas de trabajo destinado a la producción (atribuida a los varones) y a la reproducción (atribuida a las mujeres). 
de los hogares, como por un efecto de segregación ocupacional sobre las posiciones ocupacionales a las que apuntan y acceden varones y mujeres. El carácter relacional tanto del concepto de clase como del concepto de género sobre el que se han consolidado estos desarrollos, a su vez, sostiene que la integración de las mujeres a los estudios de movilidad social no sólo da cuenta de un segmento invisibilizado sino que constituye un marco de análisis más preciso para el estudio de la reproducción social. En ese sentido, tanto las diferencias entre las posiciones sociales que ocupan varones y mujeres como las distinciones entre sus trayectos intergeneracionales e intracarrera más frecuentes "hacen cambiar el equilibrio dentro del mercado y la configuración de los sistemas de oportunidades y expectativas de ambos sexos" (SALIDO CORTÉS, 1996, p. 32).

Esos debates que han circundado en las últimas décadas sobre los estudios de movilidad social han motivado no sólo la integración más frecuente de las mujeres y (un poco menos) del concepto de género en ese tipo de estudios sino el rediseño de las estrategias de construcción de las clases sociales y de análisis de los patrones de movilidad. Así, alternativas fueron creadas para la asignación de los hogares en clases, como el enfoque de dominancia (GOLDTHORPE, 1987) para identificar a los hogares en función del integrante con una posición de clase mayor, lo que hace posible la consideración de la ocupación de las mujeres en su interior. Con mayor frecuencia, a su vez, los estudios de movilidad fueron cambiando su unidad de análisis desde los hogares a los individuos, lo que ha permitido la comparación directa entre las características de las posiciones y los trayectos de varones y mujeres. Considerar a los individuos como unidad de análisis para el estudio de la movilidad social no implica que esa integración pueda tomar sólo una forma. En ese sentido, Fachelli y López-Roldán (2015) identifican tres modelos posibles para los análisis de movilidad intergeneracional en función de su consideración o no de las mujeres, y del modo en que se las considere:

- El modelo convencional que, siguiendo a Goldthorpe (1983), considera únicamente la posición social de los varones, tanto en origen como en destino.

- El modelo ampliado, que no sólo estudia los destinos sociales de los varones sino también de las mujeres, pero ambos contra la posición social de sus padres.

- El modelo integral de dominancia, que también considera los destinos de varones y mujeres pero contra un origen social construido a través de la posición más alta entre el padre y la madre.

La tesis de los autores va en línea con los argumentos feministas de finales del siglo XX, dado que consideran que "la inclusión de las mujeres tiene implicaciones tanto teóricas, pues cambia la perspectiva de conceptualización de la realidad social, como empíricas, pues los resultados de los análisis se ven modificados" (FACHELLI; LÓPEZ-ROLDÁN, 2015, p. 42-44). En su estudio con datos de 2011 para España con el objetivo de "poner de manifiesto la necesidad de tratar la sociedad en su conjunto y evidenciar las diferencias que se obtienen con relación a un análisis parcial centrado en los varones" (FACHELLI; LÓPEZ-ROLDÁN, 2015, p. 44), los autores encuentran diferencias en las tasas de movilidad al variar de modelo y considerar tanto a los varones como a las mujeres. Es así que mientras los varones presentan una reproducción mayor, las mujeres son más móviles, y se concentran en posiciones sociales de trabajo no manual o manual no calificado. Concluyen por lo tanto, en el sentido de su tesis inicial, que los resultados sobre el alcance de la movilidad social se modifican al incluir a las mujeres.

Así como en Europa, las investigaciones sobre movilidad social en América Latina respondieron a los debates anteriores integrando paulatinamente a las mujeres en sus diseños, mayoritariamente mediante la segmentación y comparación de los resultados por género. Boado y 
Solís (2016, p. 27) enfatizan en un libro sobre los patrones de movilidad intergeneracional en seis países latinoamericanos que "resulta impostergable emprender análisis empíricos que incorporen también a las mujeres" dado que sólo así es posible "conocer cuáles son las especificidades que imprime el género en la estratificación y la movilidad social en los países de la región”. Los resultados enseñan a nivel general descriptores absolutos diferentes entre los géneros, que coinciden en tasas de movilidad ligeramente superiores para las mujeres, y una segregación ocupacional que produce para las mujeres una "menor inserción en las clases manuales y agrícolas, así como una mayor concentración en la clase no manual de rutina” (BOADO Y SOLÍS, 2016, p. 124).

Unas décadas antes de esa publicación conjunta Scalon (1997) había destinado su tesis de doctorado al estudio de las tendencias y patrones de movilidad social para Brasil, con un capítulo específico para el análisis comparativo de los regímenes de movilidad de varones y mujeres. Este análisis había ido más allá de la observación de las diferencias entre las tasas absolutas de movilidad, proponiéndose contrastar la hipótesis que retoma de los trabajos de Goldthorpe sobre la similaridad entre los regímenes de movilidad relativa de varones y mujeres: "El argumento principal de los que adoptan la perspectiva convencional es que en la estructura de clases no se insertan especificidades de género que justifiquen la inclusión de información sobre las mujeres; o sea, las chances relativas de posicionamiento en la estructura social se distribuyen de tal forma con independencia del género" (SCALON, 1997, p.27; traducción propia).

Haciendo uso de los modelos loglineales para contrastar estadísticamente la fluidez social de varones y mujeres, y utilizando a su vez modelos topológicos, que permiten mapear la movilidad en zonas de similar densidad, Scalon destacó un ajuste estadísticamente significativo del modelo topológico creado tanto para los varones como para las mujeres, en el sentido de la hipótesis convencional, sin embargo, enfatizó ciertas distinciones relacionadas sobre todo con una frontera entre las posiciones manuales y no manuales más presente para las mujeres, y una frecuencia mayor de ellas en el estrato manual de baja calificación.

Son de destacar también los trabajos de Manuel Riveiro (2011, 2014 junto a GÓMEZ ROJAS, 2016), que retoma desarrollos de Gómez Rojas (2009) para Argentina y de Salido Cortés (1996) para España, por la intención del autor de trascender la segmentación de la muestra y la comparación de los resultados para varones y mujeres hacia una articulación reflexiva de la clase y el género como fuentes de desigualdad que interactúan (ejemplo de esto es su artículo de 2016 que supuso un antecedente fundamental de este trabajo por trazar una presentación aquí retomada de los diálogos conceptuales entre la teoría de género y los análisis de movilidad social). Estos esfuerzos de articulación se reflejan no sólo en enfoques teóricos con contribuciones de ambos campos de investigación sino en diseños de investigación empírica construidos para observar de manera más precisa la relación entre movilidad social y género.

Es así que en el análisis de movilidad social para Argentina Riveiro (2011) resalta que sus conclusiones no son precisas si no se observan diferencial y comparativamente los procesos de movilidad de varones y mujeres. Indica que un análisis para ese país que no distingue entre varones y mujeres pasaría por alto, por ejemplo, la feminización de las clases no calificadas tanto no manuales como manuales, así como la masculinización del estrato manual calificado y la pequeña burguesía y cómo esas diferencias, a su vez, redundan en una mayor movilidad estructural para las mujeres y en diferentes trayectorias de clase. 


\section{Consideraciones finales}

Lo revisado anteriormente sostiene entonces que la integración de las mujeres al análisis de clase y específicamente a los estudios de movilidad social se ha convertido en un requisito casi ineludible, a la vez que un desafío teórico-metodológico de muchas aristas por la complejidad que agrega al esquema teórico desde el cual se observa la reproducción social. Entre esas artistas interesa destacar, a modo de cierre, las siguientes:

- El debate entre fijar al individuo como unidad de análisis o a los hogares, lo que dificulta el estudio claro del efecto del género, dado que esta última es una variable de clasificación individual. Considerar una unidad de análisis individual, no obstante, hace más difícil la integración de variables como la conformación familiar y la división sexual del trabajo -propias del análisis de género- que se asocian con la forma en la que se distribuye el bienestar y con las decisiones que toman los sujetos sobre su vinculación con el mercado laboral.

- La consideración de, además de modos directos de clasificación de clase, formas mediadas de estratificación social, en el sentido de lo que problematiza Wright (2000), al proponer que los análisis de clase no sólo contemplen la ocupación de los individuos para asignarles una posición social (asignación directa) sino también aquellos mecanismos mediadores como el matrimonio. Esto, de acuerdo al autor, permite conceptualizar a la clase y al género no como fuentes autónomas de desigualdad sino desde su interacción: "en lugar de preguntar 'en qué clase está la persona X', deberíamos preguntar, 'cuál es la ubicación de la persona $\mathrm{X}$ dentro de una red de relaciones de clase directas y mediadas'. Si bien la pregunta es bastante poco elegante, sin embargo, identifica una dimensión crítica de la complejidad de las estructuras de clase del capitalismo contemporáneo" (WRIGHT, 2000, p.145; traducción propia).

- La cuestión de la disimilaridad entre los orígenes y los destinos (en referencia a la diferencia entre la distribución de clase de origen y la distribución de clase de destino, que hace que los marginales de las tablas de movilidad no coincidan). Esto se relaciona fuertemente con la discusión de la integración del género a los análisis de movilidad dado que la disimilaridad siempre es mayor para las mujeres por un efecto de segregación ocupacional que hace que sus estructuras de origen -que por el criterio de dominancia suelen tomar información en mayor medida de los padres que de las madres- difiera más de sus estructuras de destino que para los varones. Esto genera una movilidad forzada que aumenta sus tasas absolutas de movilidad e impacta en el estudio descriptivo de los trayectos. La distinción ofrecida al principio entre el análisis de la movilidad absoluta y el análisis de la movilidad relativa se vuelve aún más necesaria en sus implicancias al considerar el género.

Estos desafíos más que obstáculos son evidencia del camino de complejización en el estudio de la movilidad y reproducción social que se viene realizando mediante la integración del género en sus diseños y el esfuerzo de fundarlo en una discusión teórica interdisciplinaria que efectivamente permita el avance de la reflexión sociológica al respecto y, por lo tanto, pueda alimentar desde ese lugar los debates políticos y los acuerdos intersubjetivos de cooperación social. 


\section{Referencias}

ABBOTT, P.; PAYNE, G. The social mobility of women: beyond male mobility models. "Introduction: origins and destinations" y Capítulo 2: "Women's social mobility: the conventional wisdom reconsidered". Londres: The Falmer Press, 2005.

ACKER, J. Women and stratification: a case of intellectual sexism. Huber, J. Changing women in a changing society. Chicago: University of Chicago Press, 1973.

BLAU, P.; DUNCAN, O. The American occupational structure. Nueva York: The Free Press, 1978.

BOADO, M. La movilidad social en el Uruguay contemporáneo. Montevideo: IUPERJ, Ucam, UdelaR, CSIC, 2008.

BOADO, M. Modelos de movilidad social: una aproximación al funcionamiento de la desigualdad social en ciudades del Uruguay VV.AA. El Uruguay desde la sociología VIII. Montevideo: Universidad de la República, Facultad de Ciencias Sociales, 2010.

BOADO, M. Clivajes y linajes en la investigación sobre movilidad social. El Uruguay desde la sociología IX. Montevideo: Universidad de la República, Facultad de Ciencias Sociales, 2011.

BOADO, M. Reproducción y movilidad sociales en Montevideo 1996-2010. VV.AA. El Uruguay desde la Sociología XI. Montevideo: Universidad de la República, Facultad de Ciencias Sociales, 2013.

BOADO, M.; SOLÍS, P. Y sin embargo se mueve...: estratificación social y movilidad intergeneracional de clase en América Latina. Mexico D.F.: El Colegio de Mexico, Centro de estudios Espinosa Yglesias, 2016.

BUTLER, J. El género en disputa. El feminismo y la subversión de la identidad. Madrid: Paidós, 2007.

BUTLER, J. Cuerpos que importan. Sobre los límites materiales y discursivos del sexo. Buenos Aires: Paidós, 2010.

BUTLER, J.; FRASER, N. ¿Reconocimiento o redistribución? Un debate entre marxismo y feminismo. Madrid: Traficantes de sueños, 2000.

CARABAÑA, J. Las paradojas de la meritocracia. Revista de occidente. № 1 Madrid, 1980.

CARDOSO, F.; FALETTO, E. Dependencia y desarrollo en América Latina: ensayo de interpretación sociológica. México D.F.: Siglo XXI, 1987.

CORTÉS, F.; ESCOBAR, A. Movilidad social intergeneracional en el México urbano. Revista de la CEPAL, 85, 2005.

CROMPTON, R. Clase y estratificación: una introducción a los debates actuales. Madrid: Editorial Tecnos, 1994.

CROMPTON, R. Class and gender beyond the 'cultural turn'. Revista Sociología, problemas e prácticas, $n^{\circ} 42,2003$.

DELPHY, C. Women in Stratification Studies. Roberts, Helen, Doing Feminist Research. Londres: Routledge, 1981.

ENGELS, F. El origen de la familia, la propiedad privada y el Estado. Madrid: Fundación Federico Engels, 2006. 
ERIKSON, R.; GOLDTHORPE, J. The constant flux. A study of class mobility in industrial societies. Oxford: Oxford University Press, 1993.

ERIKSON, R; GOLDTHORPE, J; PORTOCARERO, L. Intergenerational Class Mobility in Three Western European Societies: England, France and Sweden. The British Journal of Sociology, Vol. 30, $\mathrm{N}^{\circ} 4$, pp. 415-441, 1979.

ESPINOZA, V.; BAROZET, E.; MÉNDEZ, M.L. Estratificación y movilidad social bajo un modelo neoliberal: el caso de Chile. Revista Lavboratorio, 25, pp. 169-191, 2013.

DALLE, P. Movilidad social intergeneracional en Argentina: oportunidades sin apertura de la estructura de clases. Revista de Ciencias Sociales, DS-FCS, vol. 28, n. 37, julio-diciembre 2015, pp. 139-165, 2015.

DE BEAUVOIR, S. El segundo sexo. Buenos Aires: Debolsillo, 2018.

DI SEGNI, S. Sexualidades. Tensiones entre la psiquiatría y los colectivos militantes. Buenos Aires: Fondo de Cultura Económica, 2013.

DO VALLE SILVA, N. Cambios sociales y estratificación en el Brasil contemporáneo (1945-1999). Franco, R; León, A; Atria, R, coords. Estratificación y movilidad en América Latina: transformaciones estructurales de un cuarto de siglo. Santiago de Chile: LOM, pp. 163-224, 2007.

FACHELLI, S.; LÓPEZ-ROLDÁN, P. ¿Somos más móviles incluyendo a la mitad invisible? Análisis de la movilidad social intergeneracional en España en 2011. Revista Española de Investigaciones Sociológicas. Madrid: Nº150 pp. 41-70, 2015.

FEDERICI, S. La reproducción de la fuerza de trabajo en la economía global y la inacabada revolución feminista. La revolución feminista inacabada. Montevideo: Minervas ediciones, 2008.

FEDERICI, S. Calibán y la bruja: mujeres, cuerpo y acumulación originaria. Buenos Aires: Tinta Limón, 2015.

FRANCO, R.; LEÓN, A.; ATRIA, R. Estratificación y movilidad social en América Latina. Transformaciones estructurales de un cuarto de siglo. Santiago de Chile: LOM Ediciones, 2007.

GANZEBOOM, H.; TREIMAN, D. The fourth generation of comparative stratification research. Quah y Sales. The international handbook of sociology (pp. 123-150). Londres: SAGE, 2000.

GANZEBOOM, H.; TREIMAN, D.; ULTEE, W. Comparative Intergenerational Stratification Researc: Three Generatons and Beyond. Annual Review of Sociology. N¹7, pp. 277-302, 1991.

GAYO, M.; MÉNDEZ, M. L. El perfil de un debate: movilidad y meritocracia. Contribución al estudio de las sociedades latinoamericanas. Franco, Rolando; León, Arturo y Atria, Raúl (coords.) Estratificación y movilidad social en América Latina. Transformaciones estructurales de un cuarto de siglo. Santiago de Chile: LOM Ediciones, 2007.

GERMANI, G. Sociología de la modernización: estudios teóricos, metodológicos y aplicados a América Latina. Buenos Aires: Paidós, 1969.

GLASS, D. Social mobility in Britain. Londres: Routledge \& Kegan Paul, 1971.

GOLDTHORPE, J. Women and class analysis: In Defence of the Conventional View. Revista Sociology, vol. 17, 1983. 
GOLDTHORPE, J. Social mobility and class structure in modern Britain. 2da edición. Oxford: Oxford University Press, 1987.

GOLDTHORPE, J. De la sociología. Números, narrativas e integración de la investigación y la teoría. Madrid: Centro de Investigaciones Sociológicas, 2010.

GOLDTHORPE, J.; MARSHALL, G. El prometedor futuro del análisis de clase: una respuesta a las críticas recientes. Revista de Sociología N³2(1), pp. 106-128, 2017.

GÓMEZ ROJAS, G.; RIVEIRO, M. Sociologando: Hacia una mirada de género en los estudios de movilidad social: interrogantes teórico-metodológicos. Boletín Científico Sapiens Research, N 1(4), 2014

HOUT, M. Mobility tables. Nueva York: SAGE, 1983.

IUTAKA, S. Estratificación social y oportunidades educacionales en tres metrópolis latinoamericanas: Buenos Aires, Montevideo y San Pablo. América Latina. Río de Janeiro: Centro Latino Americano de Investigaciones en Ciencias Sociales, 1962.

JORRAT, J. R. Estratificación social y movilidad: un estudio sobre el área metropolitana de Buenos Aires. Tucumán: Universidad Nacional de Tucumán, 2000.

JORRAT, J. Exploraciones sobre movilidad de clases en Argentina: 2003-2004. Documentos de trabajo no. 52. Buenos Aires: Instituto de Investigaciones Gino Germani, Facultad de Ciencias Sociales, UBA, 2008.

JORRAT, J. De tal padre, ¿tal hijo?: estudios sobre movilidad social y educacional en Argentina. Documentos de trabajo no. 70. Buenos Aires: Instituto de Investigaciones Gino Germani, Facultad de Ciencias Sociales, UBA, 2014.

KERBO, H. Estratificación social y desigualdad: el conflicto de clase en perspectiva histórica, comparada y global. Madrid: McGraw Hill, 2003.

KESSLER, G; ESPINOZA, V. Movilidad social y trayectorias ocupacionales en Buenos Aires.

Continuidades, rupturas y paradojas. VV.AA. Estratificación y movilidad social en América Latina: transformaciones estructurales de un cuarto de siglo - Santiago: NU. CEPAL/LOM Ediciones, 2007.

PLA, J. Trayectorias intergeneracionales de clase y marcos de certidumbre social. La desigualdad social desde la perspectiva de la movilidad. Región Metropolitana de Buenos Aires 2003-2011. Tesis de doctorado. Buenos Aires: Universidad de Buenos Aires, 2012.

PLA, J. Reflexiones sobre el uso del concepto de clase para el estudio de la movilidad social. Chávez Molina, Eduardo Desigualdad y movilidad social en el mundo contemporáneo. Buenos Aires: Imago Mundi, 2013.

PLA, J.; RODRÍGUEZ DE LA FUENTE, J.; Tendencias de movilidad social en la Argentina de las dos últimas décadas: 1995-2010. Revista Papers, 101/4, 473-502, 2016

RIVEIRO, M. Los ángeles no tienen sexo. La movilidad social sí. Buenos Aires: Instituto de Investigaciones Gino Germani, Facultad de Ciencias Sociales (UBA), 2011.

RIVEIRO, M. Apuntes críticos sobre las relaciones de género en los estudios de movilidad social intergeneracional. Revista Lavboratorio, №26, Año 16, 2016.

RUBIN, G. El tráfico de mujeres: notas sobre la economía política del sexo. Nueva Antropología, vol. VIII, núm. 30, pp. 95-145. México D.F.: Asociación Nueva Antropología A.C, 1986. 
SALIDO CORTÉS, O. La movilidad ocupacional femenina en España: una comparación por sexo. Tesis doctoral. Madrid: Universidad Complutense de Madrid, 1996.

SCALON, C. Mobilidade social no Brasil: Padroes e tendencias. Primera versión de tesis de doctorado. Río de Janeiro: IUPERJ, 1997.

SCOTT, J. El género: una categoría útil para el análisis histórico. Lamas, Marta. El género: la construcción cultural de la diferencia sexual. México D.F.: UNAM, 1996.

SOLÍS, P.; CORTÉS, F. La movilidad ocupacional en México: rasgos generales, matices regionales y diferencias por sexo. Rabell Romero, C. coord. Tramas familiares en el México contemporáneo: una perspectiva sociodemográfica. México: Instituto de Investigaciones Sociales - UNAM / El Colegio de México, pp. 395-433, 2009.

SOROKIN, P. Estratificación y movilidad social. Revista Mexicana de Sociología. Vol. 15. N1, 1953.

STANWORTH, M. Women and class analysis: a reply to John Goldthorpe. Sociology, Vol.18, № 2, 1984.

TORCHE, F.; WORMALD, G. Estratificación y movilidad social en Chile: entre la adscripción y el logro. Santiago de Chile: Cepal, Naciones Unidas, 2004.

WRIGHT, E. O. Class Counts. Student edition. Cambridge: Cambridge University Press, 2000. 